\title{
Multi-stacks of epitaxial GeSn self-assembled dots in Si: Structural analysis
}

F.Oliveira $^{\text {a,b) }}{ }^{\text {, I. A. Fischer }}{ }^{\text {a) }}$, A. Benedetti ${ }^{\text {c) }}$, M. F. Cerqueira ${ }^{\text {b) }}$, M. I. Vasilevskiy ${ }^{\text {b) }}$, S. Stefanov $^{\text {d) }}$, S. Chiussid ${ }^{\text {d) }}$, J. Schulze ${ }^{\text {a) }}$

a) Institute for Semiconductor Engineering, University of Stuttgart, 70569 Stuttgart, Germany

b) Centre of Physics, University of Minho, Campus de Gualtar, 4710-057 Braga, Portugal

c) CACTI, Univ. de Vigo, Campus Universitario Lagoas Marcosende 15, Vigo, Spain

d) Dpto. Fisica Aplicada, Univ. de Vigo, Rua Maxwell s/n, Campus Universitario Lagoas Marcosende, Vigo, Spain

\begin{abstract}
:
We report on the growth and structural and morphologic characterization of stacked layers of self-assembled GeSn dots grown on $\mathrm{Si}$ (100) substrates by molecular beam epitaxy at low substrate temperature $\mathrm{T}=350{ }^{\circ} \mathrm{C}$. Samples consist of layers (from 1 up to 10) of $\mathrm{Ge}_{0.96} \mathrm{Sn}_{0.04}$ self-assembled dots separated by Si spacer layers, $10 \mathrm{~nm}$ thick. Their structural analysis was performed based on transmission electron microscopy, atomic force microscopy and Raman scattering. We found that up to 4 stacks of dots could be grown with good dot layer homogeneity, making the GeSn dots interesting candidates for optoelectronic device applications.
\end{abstract}




\section{Introduction}

The epitaxial growth of self-assembled Ge dots on Si has been studied for several decades $[\mathbf{1}, \mathbf{2}]$. When depositing a few monolayers (ML) of Ge on a Si substrate, the lattice mismatch between Ge and Si results in the growth of Ge dots by the well-known Stranski-Krastanov mechanism [3]. The size and distribution of the Ge dots are strongly influenced by the growth parameters used. When multiple layers of Ge dots are grown, each Ge dot layer is capped by Si that forms a spacer between adjacent Ge dot layers. This Si cap also modifies dot properties such as material composition, dot geometry and strain $[4,5]$. The carrier confinement inside the Ge dots embedded in a Si matrix influences optical properties [6]. This confinement effect makes Ge dots particularly interesting for optoelectronic device applications such as photodetectors.

Recent years have seen increased efforts in the search for all-group-IV light emitters. While progress on solely Ge-based light emitters has been made [7], a large amount of research has been devoted to investigating $\mathrm{Ge}_{1-\mathrm{x}} \mathrm{Sn}_{\mathrm{x}}$ alloys as a candidate for a group-IV direct band gap material [8-15]. Lasing was observed in the partially relaxed directbandgap alloy $\mathrm{Ge}_{0.874} \mathrm{Sn}_{0.126}$ on $\mathrm{Si}[\mathbf{1 6}]$. Ge itself is an indirect band gap material whose conduction band minimum is located at the L-point. However, Ge also has a local conduction band minimum at the $\Gamma$-point whose value can be lowered by tensile strain or by introducing $\mathrm{Sn}$ to form $\mathrm{Ge}_{1-\mathrm{x}} \mathrm{Sn}_{\mathrm{x}}$ alloys. The crossover of $\mathrm{Ge}_{1-\mathrm{x}} \mathrm{Sn}_{\mathrm{x}}$ from an indirect to a direct band gap material as a function of $\mathrm{Sn}$ content has been predicted to occur for a value of $\mathrm{x}$ between 0.06 and 0.1 for the unstrained material [8, 17-19]. For pseudomorphic $\mathrm{Ge}_{1-\mathrm{x}} \mathrm{Sn}_{\mathrm{x}}$, the crossover from an indirect to a direct bandgap has been predicted to occur at $\mathrm{x} \approx 0.17[\mathbf{2 0}]$ or $\mathrm{x} \approx 0.19[\mathbf{2 1}]$.

The growth of GeSn alloys by molecular beam epitaxy remains challenging because of the large $(17 \%)$ lattice mismatch between the end member crystals and the instability of 
the diamond-cubic structure of $\alpha$-Sn above $13.2{ }^{\circ} \mathrm{C}[\mathbf{2 2}, 23]$. Recently, a lot of experimental progress [10-13] has been made towards producing GeSn alloys with $\mathrm{Sn}$ concentrations above the thermodynamic solubility of $\mathrm{Sn}$ in $\mathrm{Ge}$, which is less than 0.5 at. $\%[22,23]$.

While the above approaches were concerned with growth of bulk GeSn, several approaches have been tried to produce $\mathrm{Sn}$ or $\mathrm{Ge}_{1-\mathrm{x}} \mathrm{Sn}_{\mathrm{x}}$ nanostructures, namely: $\mathrm{Sn}$ dots were grown on $\mathrm{Ge}$ [24], $\mathrm{Ge}_{1-\mathrm{x}} \mathrm{Sn}_{\mathrm{x}}$ dots were grown on thin $\mathrm{SiO}_{2}$ layers on top of $\mathrm{Si}$ (111) substrates [25] and Sn nanostructures were also embedded into a Si or Ge matrix by annealing of SiSn or GeSn films [26]. So far, none of these approaches have lead to direct bandgap nanostructures that were embedded within Si or Ge devices. The growth of a few monolayers of $\mathrm{Ge}_{1-\mathrm{x}} \mathrm{Sn}_{\mathrm{x}}$, in conditions to reach to the formation of selfassembled $\mathrm{Ge}_{1-\mathrm{x}} \mathrm{Sn}_{\mathrm{x}}$ dots, could potentially be a means to realize direct-bandgap nanostructures embedded in Si. The present study is focused on the growth and study of multiple stacks of GeSn self assembled dots that form directly on Si (100) by depositing few monolayers of $\mathrm{Ge}_{0.96} \mathrm{Sn}_{0.04}$. Adjacent dot layers are separated by Si spacer layers. The motivation for this is twofold: on the one hand, many optoelectronic device applications need the dots fully embedded in a Si device structure such as a pin-diode; on the other hand, the optoelectronic response is, generally, enhanced using multilayer dot structures. Finally, exploring the self-assembled formation of GeSn dots with a comparatively low percentage of $\mathrm{Sn}$ in this study enables us to interpret many of our results in the context of previous research on Ge dots, and allows the design of new structures, with higher Sn amount. 


\section{Experimental details}

The samples were grown by molecular beam epitaxy (MBE) using an electron beam evaporator for Si and Knudsen cells for Ge and Sn with pyrolytic BN crucibles and a base pressure inferior than $10^{-10}$ mbar [27-29]. Si and Ge flux are monitored by a quadruple mass spectrometer that is used as a feedback to maintain the Si and Ge flux stable while the Sn flux is controlled by the cell temperature. The Si flux was calibrated by growing Si films on Si (100) wafers with a growth rate of $1 \AA$ /s. The wafer support inside the chamber creates a hard edge between the wafer and the epitaxially grown $\mathrm{Si}$ film that is used to determine the thickness of the Si film with a profilometer. The Ge flux was calibrated by growing a relaxed epitaxial Ge film on a $c$-Si (100) wafer with a slow growing rate of $\approx 0.1 \AA / \mathrm{s}$. The thickness of the film was determined by ellipsometric spectroscopy. The flux of $\mathrm{Sn}$ was calibrated by growing thin epitaxial films of $\mathrm{Ge}_{1-\mathrm{x}} \mathrm{Sn}_{\mathrm{x}}$ on Ge buffer-layers on $c$-Si (100) wafers. The absolute concentration of Sn was determined by Rutherford backscattering spectroscopy [29].

Two series with periodic multi layers samples were grown, one containing multiple layers of GeSn self-assembled dots (from now on referred to as GeSn/Si series) and the other containing multiple layers of $\mathrm{Ge}(\mathrm{Ge} / \mathrm{Si}$ series) used as a reference. Both series of samples were grown using similar conditions in order to compare/investigate the influence of Sn on the structural properties of the samples. Si (100) wafers of $100 \mathrm{~mm}$ diameter were used as substrate for all samples. The substrates were placed inside the MBE chamber and heated in situ to $900{ }^{\circ} \mathrm{C}$ for 5 minutes to remove the native $\mathrm{SiO}_{2}$ layer on its surface by thermal desorption [30]. This treatment was followed by the growth of $50 \mathrm{~nm}$ of $\mathrm{Si}$ (buffer layer), at $500{ }^{\circ} \mathrm{C}$ and with a growth rate of $1 \AA$ As, to cover remaining surface contaminants and to smoothen the surface. 
The GeSn/Si series comprises stacks of one, two, three, four and ten sheets of 5.5 ML of $\mathrm{Ge}_{0.96} \mathrm{Sn}_{0.04}$ separated by a $10 \mathrm{~nm}$ thick $\mathrm{Si}$ spacer layer (5 samples). In order to avoid/prevent the precipitation of Sn we kept a low and constant temperature of $350{ }^{\circ} \mathrm{C}$, for all the layers and samples of the $\mathrm{GeSn} / \mathrm{Si}$ series. According to literature [5], $\mathrm{Si}$ spacer layers grown at such a low temperature, when used as cap layers for Ge dots, preserve the dot geometry and reduce intermixing. The GeSn layers had a growth rate of $0.097 \AA / \mathrm{s}$, while the Si spacer layers had a growth rate of $1 \AA$.s. The composition of the 5.5 ML of $\mathrm{Ge}_{0.96} \mathrm{Sn}_{0.04}$ was achieved by a precise control of the Ge and Sn fluxes.

The reference samples of the Ge/Si series are composed of stacks of one, two, four and ten sheets of 5.5 ML of Ge separated by a $10 \mathrm{~nm}$ thick Si spacer layer (4 samples), grown also at a constant temperature of $350^{\circ} \mathrm{C}$. The Ge was grown with a growth rate of $0.087 \AA / \mathrm{s}$. The $\approx 10 \%$ difference in growth rate between both series is not expected to have an influence on the morphology of the layers. Figure 1 provides a schematic representation of the samples and fabrication steps.

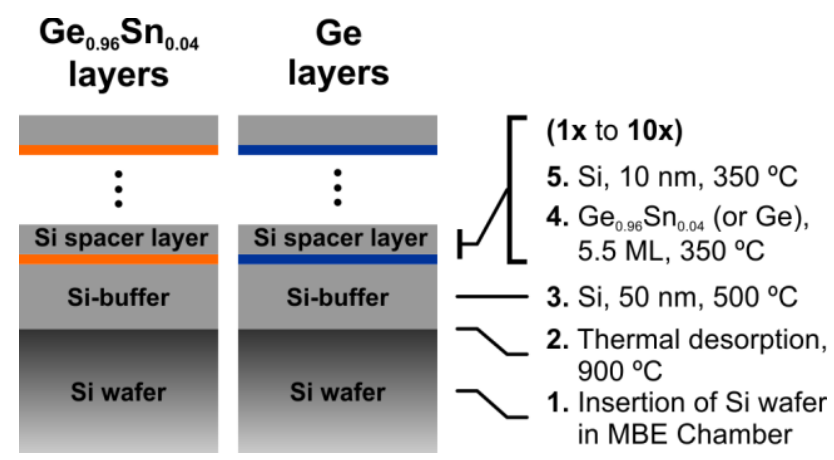

Figure 1: Illustration of the samples and respective growing steps.

The structural characterization of the samples was performed using a JEOL 2010F field emission gun Transmission Electron Microscope (TEM), working in both High 
Resolution TEM (HR-TEM), and Scanning TEM (STEM) modes. This study was complemented by atomic force microscopy (AFM) analyses and micro-Raman spectroscopy. AFM measurements were carried out with a Veeco Dimension Icon in tapping mode, in air, using Si cantilevers NT-MDT CSG10 with a cone angle lower than $22^{\circ}$. The typical curvature radius of the tip is less than $10 \mathrm{~nm}$ and a constant force of $0.1 \mathrm{~N} / \mathrm{m}$ was used. The measurements were performed for all samples of the GeSn series to obtain information about the density, shape, and size of the dots and covered area.

Raman scattering experiments were performed at room temperature in a back scattering geometry on an alpha300 R confocal Raman microscope (WITec), using a diodepumped solid state laser with a wavelength of $532 \mathrm{~nm}$ as excitation. The spot size of the excitation was $\approx 1 \mu \mathrm{m}^{2}$ with a laser power of $\approx 4 \mathrm{~mW}$ on the sample.

\section{Results and discussion}

Figure 2a (left hand side) shows a TEM cross-sectional image of the sample of the $\mathrm{GeSn} / \mathrm{Si}$ series with the largest number of dot layers $(10 \times \mathrm{GeSn})$. A TEM crosssectional image of the sample of the reference Ge/Si series with $10 \mathrm{Ge}$ layers $(10 \times \mathrm{Ge})$ is also shown for comparison (right hand side of Figure 2a). Similar images were obtained for the samples with 4 and 2 layers of GeSn or Ge. Figure 2a clearly shows some qualitative differences between the 2 samples as far as dot formation is concerned. The brighter Si spacer layers which separate the GeSn and Ge layers (darker layers) can clearly be seen in both samples, whereas dots are only observed in the GeSn/Si samples. The critical wetting layer (WL) thickness at which dot nucleation occurs is influenced by strain and substrate temperature. Since the addition of $\mathrm{Sn}$ atoms induces a larger lattice mismatch with respect to $\mathrm{Si}$ in the $\mathrm{GeSn} / \mathrm{Si}$ samples compared to the $\mathrm{Ge} / \mathrm{Si}$ ones, 
the strain in the $\mathrm{GeSn} / \mathrm{Si}$ system is higher than in the $\mathrm{Ge} / \mathrm{Si}$ system. This results in a decrease of the critical wetting layer (WL) thickness for dot formation at a given growth temperature, when $\mathrm{Sn}$ is present in the lattice. It is also known that the critical thickness of the wetting layer at which the onset of dot formation can be observed increases when the growth temperature is decreased [31]. We can conclude that the growth parameters (mainly substrate temperature) used were appropriate for the dot formation in the $\mathrm{GeSn} / \mathrm{Si}$ system but not for the Ge/Si reference sample, in which the critical WL thickness was not reached for a growth temperature of $350{ }^{\circ} \mathrm{C}$.

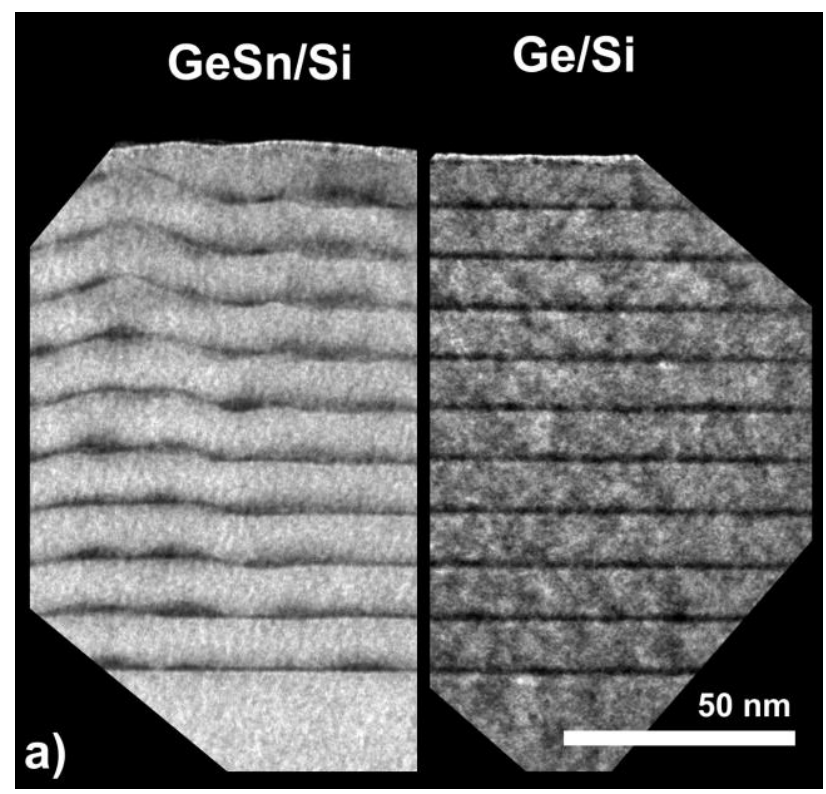



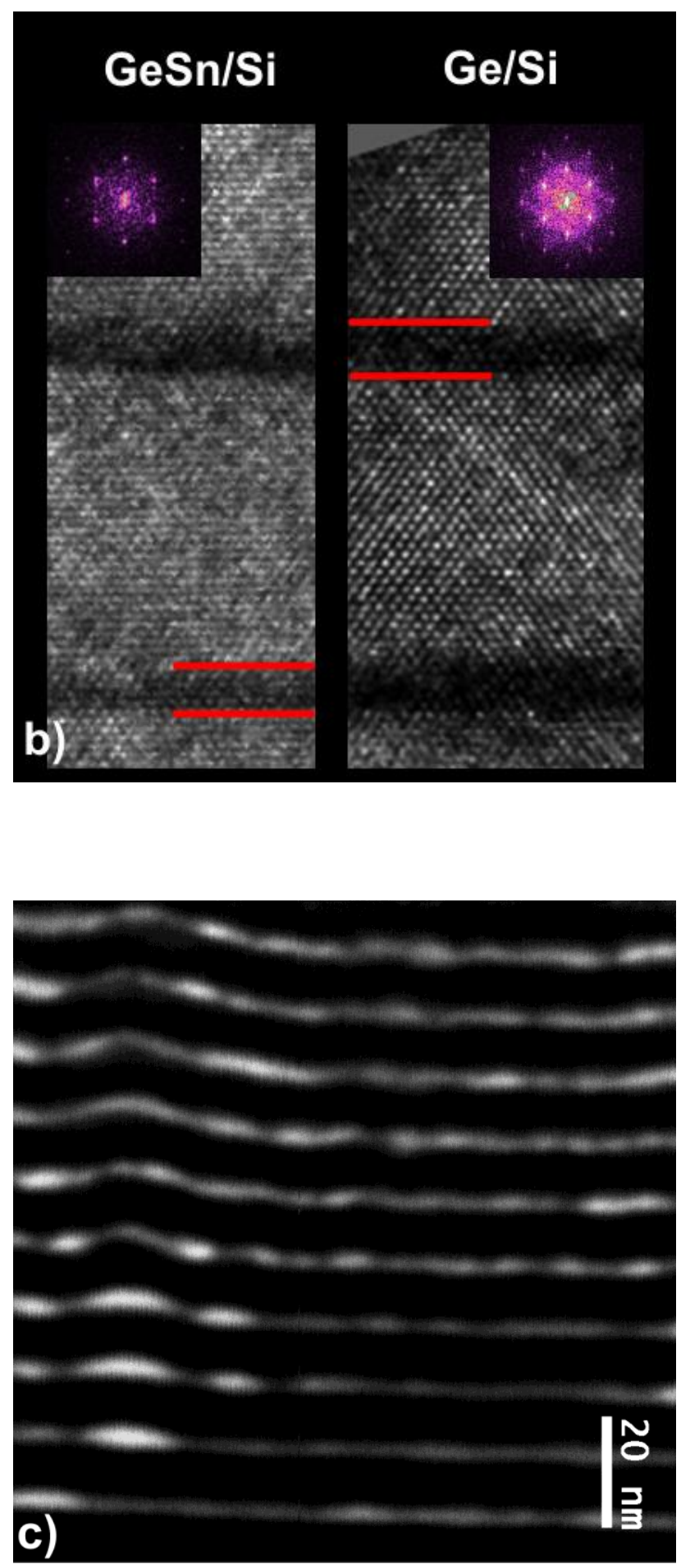

Figure 2: a) Cross-sectional TEM images of the GeSn and reference Ge samples with 10stacks. For the GeSn sample the formation of several self-assembled dots can be observed, whereas the Ge sample does not show any dots; b) Cross-sectional HR-TEM images of 4-stacks samples (GeSn and corresponding Ge reference sample), the inserts are the Fourier transform of 
the images; c) Cross-sectional STEM image of the 10-stacks GeSn ; z contrast in the image implies that darker regions correspond to lighter $\mathrm{Si}$ and brighter to heavier $\mathrm{Ge}$ and $\mathrm{Sn}$. All Figures are aligned vertically from bottom to top according to the growth direction.

It is seen from Figure 2a (left hand side) that the GeSn dots show vertical correlation between the $2^{\text {nd }}$ and the $4^{\text {th }}$ layer. Indeed, this vertical correlation of dot positions was also observed in multilayered Ge dot samples when the Si spacer layer thickness is below a critical value [32]. This correlation is attributed to the inhomogeneous strain field induced by buried dots, which will define preferential positions for dot nucleation [33]. After the $4^{\text {th }}$ layer, it is seen that the layer structure changes, namely the wetting layer is no longer planar and the dot morphology becomes different. These differences are mainly due to the occurrence of local material accumulation. Since e.g. optical characteristics are influenced by the homogeneity of the layers, such a morphological change could be detrimental to device operation.

Both the TEM (Figure 2a) and the STEM (Figure 2c) images show that all our multilayer samples $(n \times \mathrm{GeSn} / \mathrm{Si})$ have a wetting layer (WL) between dots. Based on that observation we propose that the dots are formed according to the StranskiKrastanow mechanism as is the case for pure Ge self-assembled dots.

Figure 2b shows the HR-TEM images obtained for the 4-stacks GeSn/Si sample and corresponding Ge/Si reference sample. By analysing the HR-TEM images we were able to estimate the WL thickness. For the GeSn/Si series we obtain a WL thickness of $\approx 8$ ML (measured in the vicinity of a dot in the lowest layer of the stack). It is interesting that, despite using the same growth conditions for both series, a higher WL thickness of $\approx 10 \mathrm{ML}$ was measured for the Ge/Si reference series. In both cases, the measured $\mathrm{WL}$ is thicker than the $5.5 \mathrm{ML}$ of material initially deposited, indicating that intermixing with Si occurred. The difference in WL thickness between the $\mathrm{GeSn} / \mathrm{Si}$ and the $\mathrm{Ge} / \mathrm{Si}$ 
samples can be explained by examining the case of self-assembled Ge dots grown on $\mathrm{Si}$, where dot nucleation is accompanied by the transfer of about $1 \mathrm{ML}$ of Ge from the WL to the dots [34]. One can expect a similar material transfer to take place during dot nucleation in our $\mathrm{GeSn} / \mathrm{Si}$ system, which leads to a reduction in WL thickness as compared to the Ge/Si reference system, where no such dot nucleation has occurred.

The TEM and HR-TEM images do not reveal any segregation or precipitation of Sn. The Fourier Transform for the HR-TEM contains similar information to a X-ray diffractogram. The inset in Figure $\mathbf{2 b}$ shows the Fourier Transform of the HR-TEM image for the GeSn/Si and Ge/Si layers showing a diamond structure for both samples, as well as good crystallinity. The addition of Sn can be expected to influence surface processes and, therefore, the composition of the dots. Brehm et al [35] have shown that the composition of Ge dots strongly vary along the growth direction. In particular, even for low growth temperatures, some intermixing between $\mathrm{Ge}$ and $\mathrm{Si}$ is always present. The STEM analysis of the $\mathrm{GeSn} / \mathrm{Si}$ sample (Figure 2c) reveals that a higher concentration of GeSn is present in the dots than in the WL, and that the centre of the dot also has a higher concentration of GeSn than the border. From Raman measurements that will be discussed below we can infer that intermixing with $\mathrm{Si}$ also takes place in our samples. A particularly interesting question is if and how Sn-rich regions within the dots are formed. Because of the low concentration of Sn per stack it is not possible to obtain that information from the HR-TEM and STEM images. A composition analysis with sub-nanometre resolution would, however, be useful to investigate this position-dependent intermixing.

The AFM images $\left(250 \times 250 \mathrm{~nm}^{2}\right)$ of all the $\mathrm{GeSn} / \mathrm{Si}$ samples reveal that the topmost layer of each sample contains dots, as seen in Figure 3a. However, both the density and 
the shape of the dots change with the number of sample stacks. For the 1-stack sample all the dots have a nearly spherical shape, changing to a truncated quadrangular pyramid with the base aligned along the (110) direction as the number of stacks increases (see Figure $3 \mathbf{a}$ and $\mathbf{3 b}$ ). In the uppermost layer of the 2-stack sample, the dots' shape becomes less spherical (compared to the 1-stack sample) and in the 3-stack sample dots with round and quadrangular pyramid shapes can be seen to coexist in their uppermost layer. The 4-stack sample has only quadrangular pyramid shaped dots in the uppermost layer, and the 10-stack sample contains in addition fewer large dots. A more detailed analysis of the dots with truncated quadrangular pyramid shape observed in the uppermost layer of the 4-stacks $\mathrm{GeSn} / \mathrm{Si}$ sample shows than the dots have a sidewalls with angles in the range $17-19^{\circ}$ corresponding to (130) crystal planes (see Figure $3 \mathbf{b}$ ). We note that this differs from the $11^{\circ}$ slope of the $\{105\}$ side facets that are observed in pyramidal "hut cluster" Ge islands on Si [36-38]. However, in our system the result of the shape of the dots on the surface is not only a consequence of the top most layer but also the accumulation of material below, as evidenced by the change in shape of the dots in the topmost layer as a function of the total number of dot layers below. 

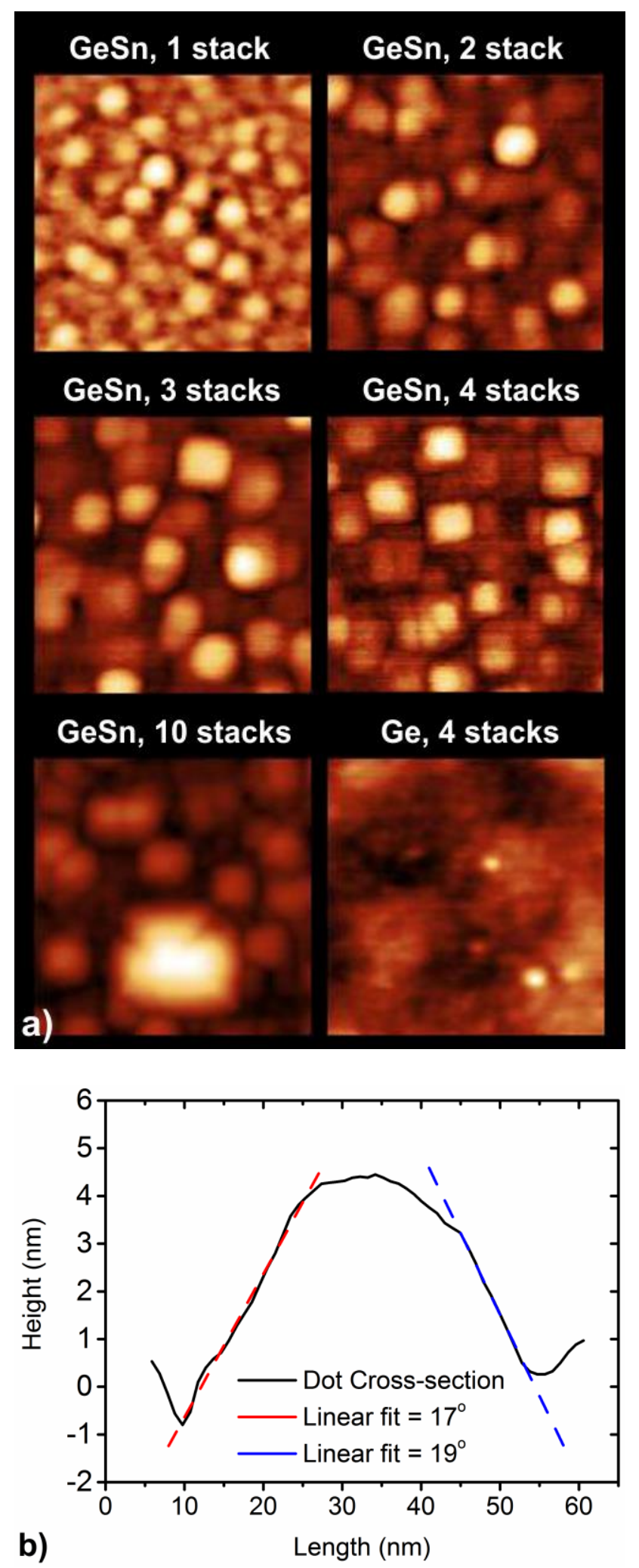


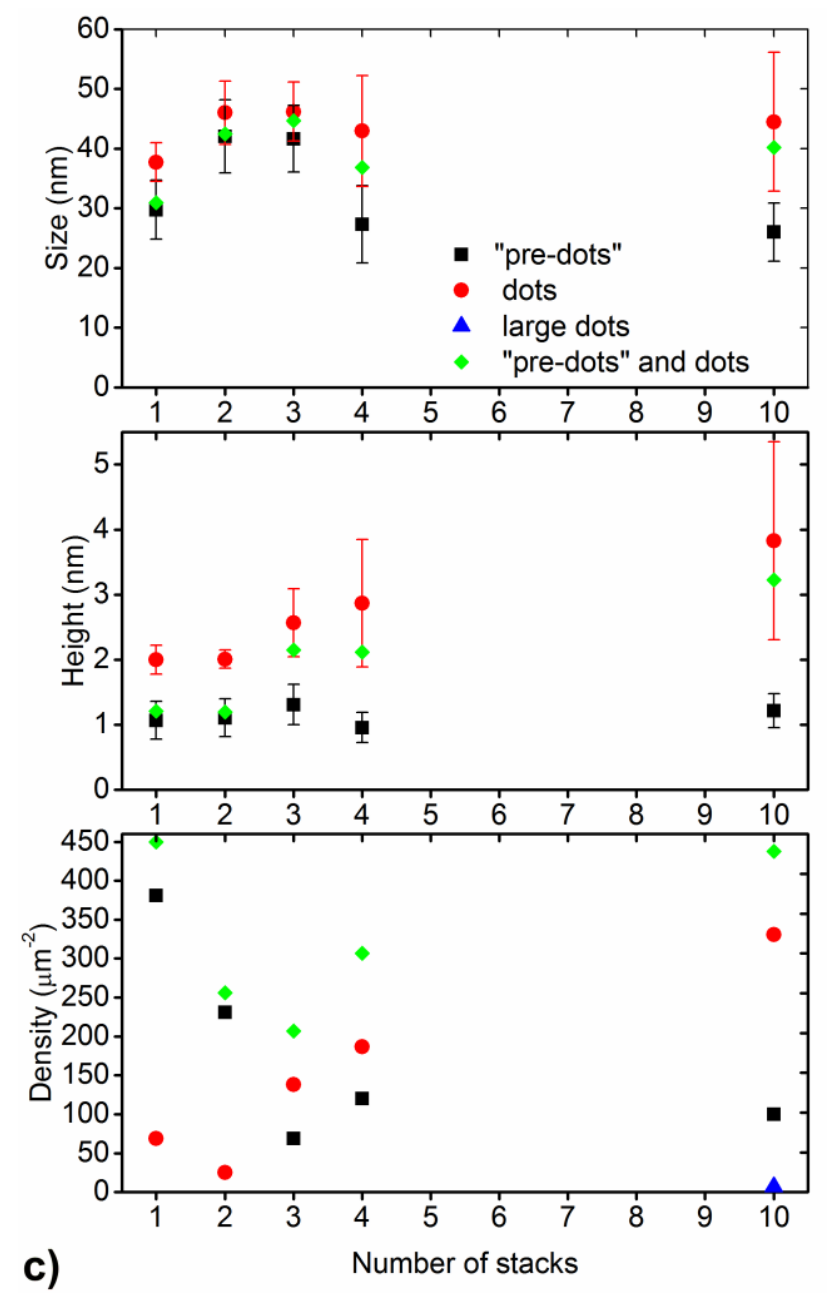

Figure 3: a) AFM images with $250 \times 250 \mathrm{~nm}^{2}$ of the surface of the GeSn samples (1- to 10stacks) and the Ge reference sample with 4-stacks. A transition from round dots to square pyramid like dots with the top truncated is seen with the increase of the number of stacks in the GeSn samples. Domes are visible only in the 10 -stacks GeSn sample. The Ge reference sample shows a much lower density and smaller size of dots than the ones present in the GeSn samples. The height (provided by the colour) is not normalized between images; b) Dot height as a function of length (cross-section) of 4-stacks GeSn sample and correspondent linear fit which give an angle coincident to (130) Miller planes; c) Variation, with respect to the number of stacks, of the density, average size and height of dots, "pre-dots" and large dots. 
The AFM image of the 10-stack sample shows the presence of few large dots (see Figure 3a) with a height of $\approx 15 \mathrm{~nm}$. As can be inferred from the STEM image of the 10-stack sample (Figure 2c), the larger dots seen by AFM on the top most layer do not form as GeSn-rich dots within one layer, but are the result of local material accumulation in the layers below. Excluding these large dots, the rest of the dots in the layers can be classified according to their height (and irrespective of their shape) in two categories: (i) "pre-dots", with a height between one and three lattice constants (in our case, 4 to 12 ML for diamond lattice) and (ii) "dots", which are higher than three lattice constants. The "pre-dots" observed in our samples do not necessarily have a square base, differing therefore from the reported "pre-pyramids" observed as the first stage of morphological evolution of Ge dots identified in refs. [38, 39]. This difference can either be attributed to the very low growth temperature $\left(350^{\circ} \mathrm{C}\right.$ compared to a minimum growth temperature of $550{ }^{\circ} \mathrm{C}$ used in [38]) or to the presence of $\mathrm{Sn}$.

The dot density and size was estimated from the AFM measurements. Figure $3 \mathbf{c}$ shows, for all GeSn samples, the average size (diameter for round dots and side length for pyramid-like ones), height and the density of pre-dots and dots per $\mu \mathrm{m}^{2}$, with the respective standard deviations, as a function of the stacks number in the sample. It can be seen from Figure 3c that the number of pre-dots (black squares) decreases as the number of stacks increases, reaching a minimum density for the 3-stack sample. For a higher number of stacks their density slightly increases and seems to stabilize (the 4and 10-stack samples have similar dot densities). The size first increases with the number of stacks, reaching a maximum at 2- and 3- stacks, then decreases slightly and stabilizes for larger numbers of stacks (Figure 3c). Concerning the dots (red circles) it is seen that their density increases as the number of stacks increases $(n \geq 2)$, and that from layer 2 onward, the dot height increases with the number of stacks, while the 
height of the pre-dots remains almost constant (consequence of definition of pre-dot). This behaviour of the dot height is consistent with the observation from the TEM study (Figure 2a), where we observe vertical dot alignment between the $2^{\text {nd }}$ to $4^{\text {th }}$ layer, which can cause a progressive height increase for the dots as observed by the AFM measurement analysis. Again, we observe first an increase in dot size with increasing number of stacks and then a stabilization of the size for 2 or more layers.

It is reported that $\mathrm{Ge}$ dots of multi-layers of $\mathrm{Ge}$ in $\mathrm{Si}$ can acquire a lateral ordering of $\mathrm{Ge}$ islands [40]. We performed an analysis of the nearest-neighbor angular distribution function by computing the angles obtained from the lines connecting the center of each island its nearest neighbors with respect to (110) direction, for several hundred islands in each sample of the GeSn/Si series and found a homogeneous distribution of angles, which means lateral ordering does not take place in any of our samples.

Figure 4a shows the Raman spectra for all $\mathrm{GeSn} / \mathrm{Si}$ samples and a $c$-Si reference spectrum for comparison. All the samples exhibit the prominent $\mathrm{Si}-\mathrm{Si}(\Gamma)$ mode at 520.8 $\mathrm{cm}^{-1}$ as well as Si related two-phonon modes, namely: 2TA $(\Sigma)$ at $433 \mathrm{~cm}^{-1}$ and 2TA (X) at $302.5 \mathrm{~cm}^{-1}$. In addition to the Si related modes, the Raman spectra of the GeSn/Si samples show the characteristic Ge-Ge band at $300.7 \mathrm{~cm}^{-1}$ and also a mode at $\approx 418 \mathrm{~cm}^{-}$ ${ }^{1}$ which is the signature of mixed Ge-Si bonds [41-43]. The presence of Sn was not seen in our samples, since no Sn-related modes were detected (Ge-Sn, Si-Sn or Sn-Sn modes [44-46]). The absence of Sn related modes can be attributed to the low amount of Sn (less than 0.3 at. \% in volume) corresponding to a low overall number of Ge-Sn, Sn-Sn and $\mathrm{Si}-\mathrm{Sn}$ bonds in our samples. 

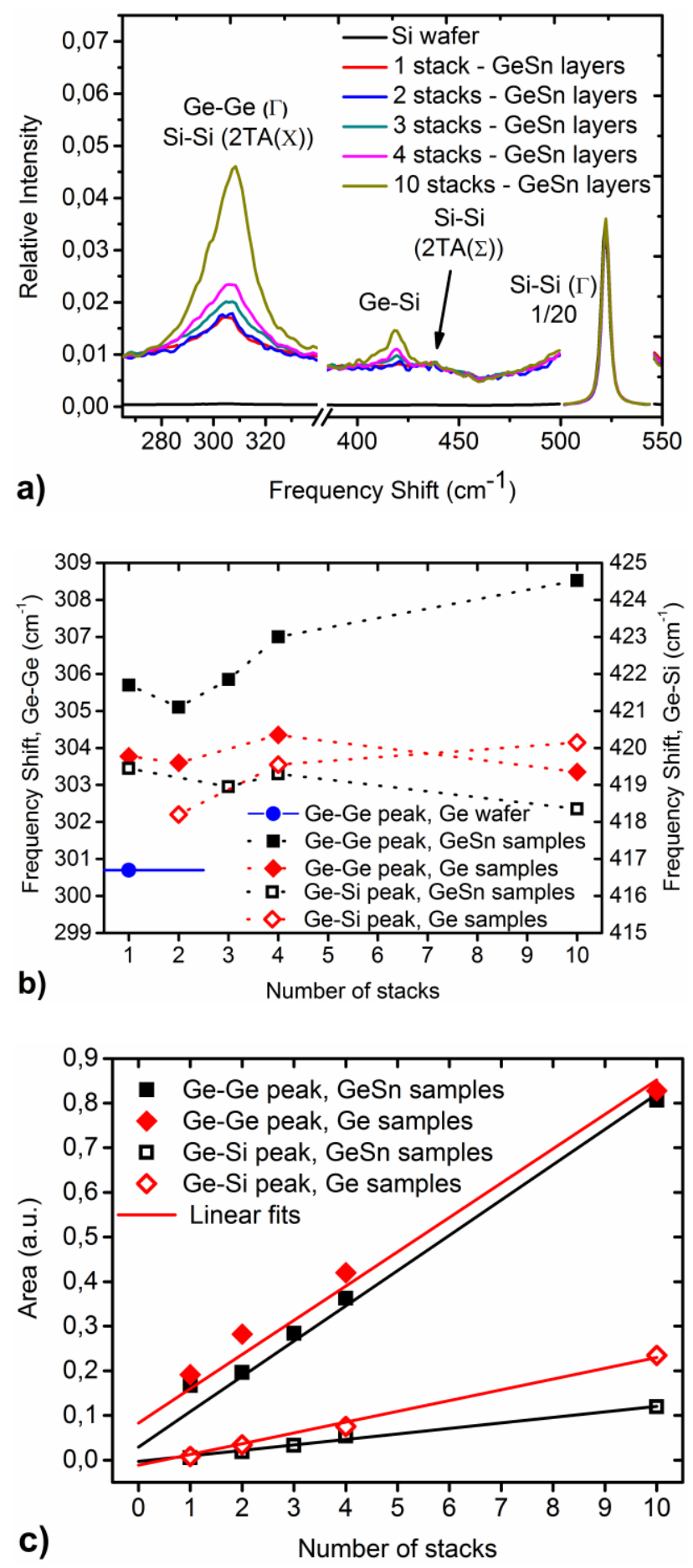

Figure 4: a) Raman spectra of the GeSn samples and $c$-Si (100) for comparison; b) Peak frequency position as a function of the number of periods/stacks of Ge-Ge and Ge-Si Raman peaks for the GeSn self-assembled dots samples and corresponding Ge references samples after Si Raman spectrum subtraction; c) Peak area and linear fit variation as a function of the number of periods/stacks for the Ge-Ge and Ge-Si Raman peaks of the GeSn self-assembled dots 
samples and Ge samples after the Si Raman spectrum subtraction. The results were obtain with $532 \mathrm{~nm}$ excitation and normalized by the Si-Si mode peak intensity.

The peak position (frequency shift) and the integrated intensity/area of the Raman band are parameters that allow to compare the different samples, since changes in the peak position are related to strain and alloy composition, while the peak area is related to the composition and short-range order of the alloy $[\mathbf{4 2}, \mathbf{4 3}]$. The observed peak position of the $\mathrm{Si}-\mathrm{Si}(\mathrm{Ge}-\mathrm{Ge})$ mode in the studied samples is shifted relatively to the position of the corresponding mode in the $\mathrm{Si}(\mathrm{Ge})$ bulk sample. For this kind of materials, a shift in the peak position is usually related with composition and strain as follows: tensile strain or the influence of heavier atoms in the lattice shifts the peak position to lower frequencies (red shift); while lighter atoms or compressive strain shifts the peak to higher frequencies (blue shift).

Concerning the $\mathrm{Si}-\mathrm{Si}$ (LO-TO) mode in the Raman spectra of our samples, it is not possible to distinguish the contributions from the Si substrate, the buffer layer and the spacers (Figure 4a), implying that the $\mathrm{Si}$ is crystalline everywhere in our samples.

The Ge-Ge and the Si-Si 2TA modes are both present in our samples at spectral positions that nearly coincide. In order to extract information concerning the Ge-Ge mode we subtracted the Si reference spectrum from the analysed spectra [41] removing the contribution of the $\mathrm{Si}-\mathrm{Si}(2 \mathrm{TA}(\mathrm{X}))$ mode. By fitting the Raman spectra it is possible to obtain the peak position of the $\mathrm{Si}-\mathrm{Si}$, Ge-Ge and Ge-Si modes. Figures $\mathbf{4 b}$ and $\mathbf{4 c}$ show the dependence of the peak positions and the band areas of these modes upon the number of $\mathrm{GeSn} / \mathrm{Si}$ and $\mathrm{Ge} / \mathrm{Si}$ stacks.

The peak positions of the Ge-Si mode for the samples ( $\mathrm{GeSn} / \mathrm{Si}$ and $\mathrm{Ge} / \mathrm{Si}$ series) lie between 416 and $419 \mathrm{~cm}^{-1}$, higher than the $410 \mathrm{~cm}^{-1}$ value reported for unstrained Ge-Si alloys for any Ge content [42]. Such a high blue shift of the $\mathrm{Si}-\mathrm{Ge}$ mode peak in $\mathrm{Ge} / \mathrm{Si}$ 
quantum dot samples has been observed before $[\mathbf{4 1 , 4 7 ]}$ and is attributed to strain [41]. Note that the effect of the number of stacks and the presence of Sn on the position of this Ge-Si mode is small $\left(\approx 1 \mathrm{~cm}^{-1}\right.$ for GeSn samples and $\approx 2 \mathrm{~cm}^{-1}$ for the Ge reference samples).

From Figure $\mathbf{4 b}$ it is seen that the Ge-Ge peak position for all samples is also shifted to higher frequencies as compared to that in bulk Ge. Furthermore, it shifts with the number of stacks, which is not the case for the Ge-Si mode. Indeed, in the system studied here a shift in the peak position is expected because the Ge-Ge mode peak position is affected by the presence, in the lattice, of heavier atoms ( $\mathrm{Sn}$, which causes a red shift) and lighter atoms ( $\mathrm{Si}$, which causes a blue shift) and also by tensile strain along the axis normal to the surface (causing a blue shift as well). However, the shift observed for this mode in the $\mathrm{GeSn} / \mathrm{Si}$ samples is quite large, in comparison with the bulk material $\left(\approx 5 \mathrm{~cm}^{-1}\right.$ for 1 -stack sample and $\approx 8 \mathrm{~cm}^{-1}$, for the 10 -stack sample) and with the Ge/Si reference samples $\left(\approx 3 \mathrm{~cm}^{-1}\right.$ for 1 stack sample and $\approx 2.5 \mathrm{~cm}^{-1}$, for the 10 stack sample). A possible explanation is that Sn preferably intermixes with Ge rather than with $\mathrm{Si}$ and the strain is introduced mostly in the Ge-rich regions.

Finally, the peak area increases proportionally to the number of stacks for both Ge-Ge and Ge-Si modes as shown in Figure 4c, which indicates that the layers contribute almost evenly to the scattering by these phonons involving vibrations of $\mathrm{Ge}$ atoms. The Raman spectra of the GeSn/Si samples with 2-, 3-, 4- and 10-layers of GeSn dots, thus, include contributions from all layers. We nonetheless find that the number of stacks has a marked influence on the position of the Ge-Ge peak in the $\mathrm{GeSn} / \mathrm{Si}$ samples. It seems reasonable to assume that there is not only a morphological evolution of the dot shape with the increase of the number of layers, as evidenced by AFM and TEM 
measurements, but also a variation of composition and strain between the different dot layers, which could explain this shift in Ge-Ge peak.

\section{Conclusions}

In summary, we grew samples with 1 to 10 stacks, each consisting of $5.5 \mathrm{ML}$ of $\mathrm{Ge}_{0.96} \mathrm{Sn}_{0.04}$ followed by $10 \mathrm{~nm}$ of $\mathrm{Si}$, in order to obtain self-assembled GeSn dots embedded in $\mathrm{Si}$ and analysed their structure. We showed that it is possible not only to produce self-assembled GeSn dots in this way but, even more importantly from the technological point of view, to overgrow the GeSn dots using Si substrates making their incorporation into Si-based devices possible. The structural characterization revealed that in some aspects, such as the dot shape, GeSn/Si samples seem to differ from their Ge counterparts. Future studies will be focused on understanding in more detail the effect of Sn on the growth kinetics of GeSn dots.

Fabricating self-assembled GeSn dots on Si could be a way towards producing directbandgap nanostructures that can be embedded in Si. To be able to do that, however, GeSn dots with higher Sn content are needed and will be investigated in order to explore the potential of such GeSn nanostructures for optoelectronic devices applications.

\section{Acknowledgements}

This work was partly supported by the Portuguese Foundation for Science and Technology (FCT) through Strategic Project PEst-C/FIS/UI0607/2013 and PhD Fellowship (F. Oliveira). 


\section{Bibliography:}

[1] Y.-W. Mo, D. E. Savage, B. S. Swartzentruber and M. G. Lagally, Phys. Rev. Lett. 65, $1020(1990)$

[2] D. J. Eaglesham and M. Cerullo, Phys. Rev. Lett. 64, 1943 (1990)

[3] I. N. Stranski and Krastanow, Sitzungsber. Wien. Akad. Wiss Math.-Nat. Kl, IIB 146, $797(1938)$

[4] J. Stangl, V. Holý, and G. Bauer, Rev. Mod. Phys. 76, 725 (2004)

[5] M. Brehm, M. Grydlik, H. Groiss, F. Hackl, F. Schäffler, T. Fromherz and G. Bauer, J. Appl. Phys. 109, 123505 (2011)

[6] K. L. Wang, Dongho Cha, Jianlin Liu and C. Chen, Proc. IEEE 95, 1866 (2007)

[7] J. Liu, L. C. Kimerling, and J. Michel, Semicond. Sci. Technol. 27, 094006 (2012)

[8] J. Kouvetakis, J. Menendez and A. V. G. Chizmeshya, Annu. Rev. Mater. Res. 36, 497 (2006)

[9] M. Oehme, M. Schmid, M. Kaschel, M. Gollhofer, D. Widmann, E. Kasper and J. Schulze, Appl. Phys. Lett. 101, 141110 (2012)

[10] H. H. Tseng, K. Y. Wu, H. Li, V. Mashanov, H. H. Cheng, G. Sun and R. A. Soref, Appl. Phys. Lett. 102, 182106 (2013)

[11] O. Nakatsuka, Y. Shimura, W. Takeuchi, N. Taoka and S. Zaima, Solid State Electronics 83, 82 (2013)

[12] B. Vincent, F. Gencarelli, H. Bender, C. Merckling, B. Douhard, D. H. Petersen, O. Hansen, H. H. Henrichsen, J. Meersschaut, W. Vandervorst, M. Heyns, R. Loo and M. Caymax, Appl. Phys. Lett. 99, 152103 (2011)

[13] S.Wirths, Z. Ikonic, A. T. Tiedemann, B. Holländer, T. Stoica, G. Mussler, U. Breuer, J. M. Hartmann, A. Benedetti, S. Chiussi, D. Grützmacher, S. Mantl and D. Buca, Applied Physics Letters 103, 192110 (2013) 
[14] M. Rojas-Lópes, H. Navarro-Contreras, P. Desjardins, O. Gurdal, N. Taylor, J. R. A. Carlsson and J. E. Greene, Journal of Applied Physics 84, 2219 (1998)

[15] P. Desjardins, T. Spila, O. Gürdal, N. Taylor and J. E. Greene, Physical Review B 60, 15993, (1999)

[16] S. Wirths, R. Geiger, N. von den Driesch, G. Mussler, T. Stoica, S. Mantl, Z. Ikonic, M. Luysberg, S. Chiussi, J. M. Hartmann, H. Sigg, J. Faist, D. Buca and D. Grützmacher, Nature Photonics, 9, 88-92 (2015)

[17] G. He and H. A. Atwater, Phys. Rev. Lett. 79, 1937 (1997)

[18] H. Lin, R. Chen, W. Lu, Y. Huo, T. I. Kamins and J. S. Harris, Appl. Phys. Lett. $100,102109(2012)$

[19] S. Gupta, B. Magyari-Kope, Y. Nishi and K. C. Saraswat, J. Appl. Phys. 113, $073707(2013)$

[20] A. A. Tonkikh, C. Eisenschmidt, V. G. Talalaev, N. D. Zakharov, J. Schilling, G. Schmidt and P. Werner, Appl. Phys. Lett. 103, 032106 (2013)

[21] L Jiang, J D Gallagher, C L Senaratne, T Aoki, J Mathews, J Kouvetakis and J Menéndez, Semicond. Sci. Technol. 29, 115028 (2014)

[22] O. Gurdal, P. Desjardins, J. R. A. Carlsson, N. Taylor, H. H. Radamson, J.-E. Sundgren and J. E. Greene, Journal of Applied Physics 83, 162 (1998)

[23] M. Bauer, J. Taraci, J. Tolle, A. V. G. Chizmeshya, S. Zollner, David J. Smith, J. Menendez, Changwu Hu and J. Kouvetakis, Applied Physics Letters 81, 2992 (2002)

[24] W. Dondl, P. Schittenhelm and G. Abstreiter, Thin Solid Films 294, 308 (1997)

[25] Y. Nakamura, A. Masada, S.-P. Cho, N. Tanaka and M. Ichikawa, J. Appl. Phys. $102,124302(2007)$

[26] R. Ragan, K. S. Min and H. A. Atwater, Mater. Sci. Eng. B 87, 204 (2001) 
[27] M. Oehme, J. Werner, O. Kirfel and E. Kasper, Applied Surface Science 254, $6238(2008)$

[28] M. Oehme, J. Werner, M. Jutzi, G. Wöhl, E. Kasper and M. Berroth, Thin Solid Films 508, 393 (2006)

[29] M. Oehme, E. Kasper and J. Schulze, ECS Transactions 50, 583 (2012)

[30] E. Kasper, M. Bauer and M. Oehme, Thin Solid Films 321, 148 (1998)

[31] R. Bergamaschini, M. Brehm, M. Grydlik, T. Fromherz, G. Bauer and F. Montalenti, Nanotechnology 22, 285704 (2011)

[32] O. G. Schmidt, O. Kienzle, Y. Hao, K. Eberl and F. Ernst, Appl. Phys. Lett. 74, $1272(1999)$

[33] G. Springholz, in Handbook of Self Assembled Semiconductor Nanostructures for Novel Devices in Photonics and Electronics, edited by M. Henini (Elsevier, 2008), p. 1

[34] M. Brehm, M. Grydlik, H. Groiss, F. Hackl, F. Schäffler, T. Fromherz and G. Bauer, J. Appl. Phys. 109, 123505 (2011)

[35] M Brehm, M Grydlik, F Hackl, E Lausecker, T Fromherz and G Bauer, Nanoscale Research Letters 5, 1868 (2010)

[36] D. E. Jesson, K. M. Chen and S. J. Pennycook, MRS Bulletin 21, April, p: 31, (1996)

[37] B.Voigtländer, Surface Science Reports43, 127 (2001)

[38] A. Rastelli, M. Stoffel, U. Denker, T. Merdzhanova and O. G. Schmidt, Phys. Status Solidi A 203, 3506 (2006)

[39] A. Vailionis, B. Cho, G. Glass, P. Desjardins, David G. Cahill, and J. E. Greene, Physical Review Letters 85, 3672 (2000) 
[40] G. Capellini, M. De Seta, F. Evangelisti, V. A. Zinovyev, G. Vastola, F. Montalenti, and Leo Miglio, Phys. Rev. Lett. 96, 106102 (2006)

[41] P. H. Tan, K. Brunner, D. Bougeard and G. Abstreiter, Phys. Rev. B 68, 125302 (2003)

[42] O. Pagès, J. Souhabi, V. J. B. Torres, A. V. Postnikov and K. Rustagi, Physical Review B 86, 045201 (2012)

[43] A. S. Vasin, O. V. Vikhrova, and M. I. Vasilevskiy, J. Appl. Phys. 115, 143505 (2014)

[44] V. R. D’Costa, J. Tolle, C. D. Poweleit, J. Kouvetakis and J. Menéndez, Phys. Rev. B 76, 035211 (2007)

[45] M. Iliev, M. Sinyukov and M. Cardona, Physical Review B 16, 5350 (1977)

[46] J.-H. Fournier-Lupien, S. Mukherjee, S. Wirths, E. Pippel, N. Hayazawa, G. Mussler, J. M. Hartmann, P. Desjardins, D. Buca, and O. Moutanabbir, Appl. Phys. Lett. 103, 263103 (2013)

[47] A. I. Yakimov, A. V. Dvurechenskii, A. I. Nikiforov, A. A. Bloshkin, A. V. Nenashev and V. A. Volodin, Physical Review B 73, 115333 (2006) 